\title{
A Horizontal Powder Injector for W7-X
}

\author{
Alexander Nagy ${ }^{\mathrm{a}}$, A. Bortolon ${ }^{\mathrm{a}}$, D. Gates ${ }^{\mathrm{a}}$, E. Gilson ${ }^{\mathrm{a}}$, C. Killer ${ }^{\mathrm{b}}$, T. Klinger ${ }^{\mathrm{b}}$, R. Lunsford ${ }^{\mathrm{a}}$, \\ R. Maingi ${ }^{\mathrm{a}}$, D. Mansfield ${ }^{\mathrm{a}}$, D. Mauzey ${ }^{\mathrm{a}}$, R. Nazikian ${ }^{\mathrm{a}}$, L. Roquemore ${ }^{\mathrm{a}}$, E. Wolfe ${ }^{\mathrm{a}}$, \\ ${ }^{a}$ Plasma Physics Laboratory, 100 Stellerator Road, Princeton, N.J. 08543 \\ ${ }^{b}$ Max-Planck-Institut für Plasmaphysik, Teilinstitut Greifswald, Wendelsteinstraße 1, D-17491 Greifswald
}

\begin{abstract}
Injection of low-Z powders into fusion plasma has been used to improve wall conditions, similar to the standard boronization process using diborane. Powder injection has the advantage of being much simpler, non-toxic, and efficient. The W7-X stellarator is planning on utilizing powder injection in long pulse discharges; a proof-of-principle test for horizontal injection into the plasma was conceived, a Probe Mounted Particle Injector (PMPI). The PMPI concept is developed using a polyetheretherketone (PEEK) paddle wheel hub that is driven by a piezo motor, due to the high magnetic fields, that rotates at $100 \mathrm{deg} / \mathrm{s}$. This small PMPI fits into an envelope of $120 \mathrm{~mm}$ diameter x $150 \mathrm{~mm}$ long, a standard size for Multi-Purpose Manipulator (MPM). The device is housed in a carbon cup mounted on a retractable probe that can be placed near the plasma edge, enabling powder injection $\sim 4-8 \mathrm{~cm}$ radially into the boundary plasma. The feed for the paddle is via piezo electric actuator that vibrates a funnel filled with powder into a trough for the paddle to push. The 8 paddle arms, $35 \mathrm{~mm}$ long and $10 \mathrm{~mm}$ wide, are made from $0.38 \mathrm{~mm}$ thick PEEK which drag slightly along the powder-filled trough bottom, becoming a spring-loaded paddle which accelerates the powder upon release. Design challenges are the high ambient magnetic field, vacuum compatible materials, high temperature environment, limited rotary-drive options, and compact space. The design and testing of this new device will be presented.
\end{abstract}

Keywords: impurity, powder, injection, horizontal, piezo feeder, boronization

\section{Introduction}

Deposition of boron coatings on plasma facing components (PFC) is a common and effective technique to condition plasma devices for fusion research. Boronization is typically accomplished through long hydrogen or helium glow discharges enriched with diborane gas. Glow discharge boronization is typically one or more days to complete and, due to diborane's health hazards, requires site evacuation. Furthermore, it must be repeated periodically to replace the coating worn off by plasma operations. As a reference, in the DIII-D tokamak, boronization is performed approximately every 4 to 6 weeks during the run campaign, with its beneficial effects lasting approximately 5 days operations, i.e. 150 shots [1].

Recently, injection of boron powder into fusion plasmas has been investigated in multiple fusion devices as a method to boronize the plasma facing components [1]. Initial studies on ASDEX-Upgrade (AUG) and DIII-D indicate that B powder, vertically dropped into tokamak plasma discharges was instrumental to successfully produce and maintain low-density plasmas [2]. The initial results suggest that boron powder injection into hot fusion plasmas might be used to replenish boron coatings and ensure more consistent wall conditions throughout the run without the associated safety and schedule penalties.

These promising observations motivated experiments to test boronization through powder injection on the W7-X with first experiments planned for September 2018. These will be executed using the compact, probe- mounted powder injector (PMPI), which will be installed on the reciprocating multi-purpose manipulator (MPM) and is the subject of this paper. The PMPI provides a metered method to inject boron carbide powder (254 microns particle average size) into the W7-X plasma edge, a minimum distance of $80-160 \mathrm{~mm}$ from the PMPI plasma facing end. This device fits within the MPM probe head envelope 120mm diameter x $150 \mathrm{~mm}$ long. See figure 1 for the MPM overall view, and figure 2 for pictures of the PMPI assembly. If the first experiments successfully confirm the potential of powder injection for conditioning W7-X, then a vertical drop unit, with multiple larger reservoirs would be considered, similar to other units presently deployed on AUG, DIII-D, EAST and KSTAR and [3].

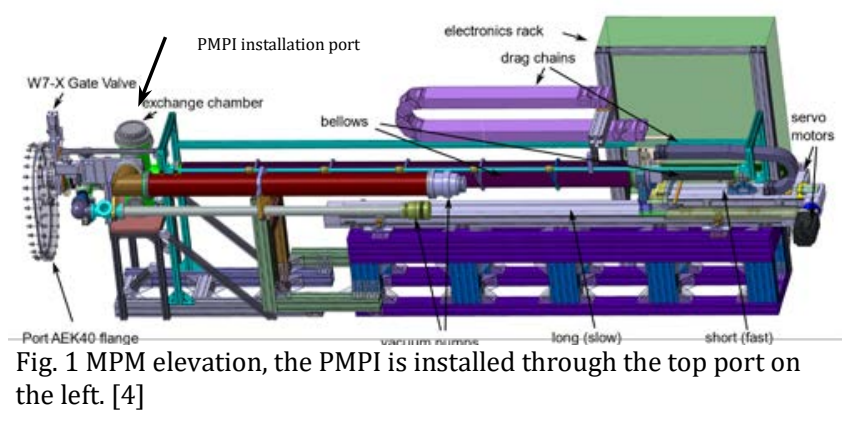



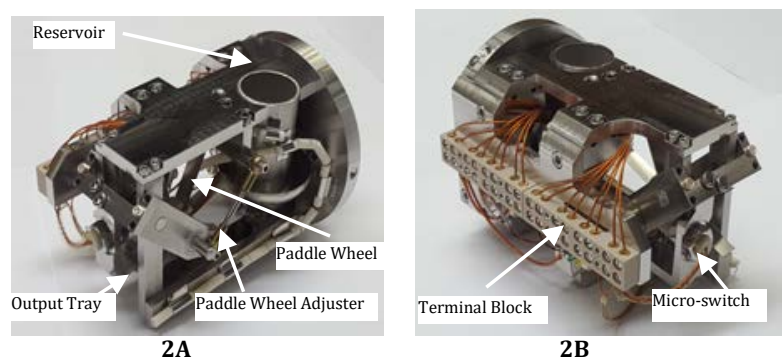

Fig. 2 Picture 2A shows the circular powder reservoir and paddle wheel (shown with SS paddles), 2B shows the wiring terminal block, and switch used for rotation monitoring.

\section{Design Details}

\subsection{Design Requirements}

The PMPI environment requires a horizontal injection of powder into the W7-X plasma. The paddle wheel concept was chosen because it could launch powder into the plasma on a continuous basis at variable rates and slow rotational speeds e.g. $<1 \mathrm{~Hz}$. The paddles scrape the bottom of the powder delivery tray and spring release off the bottom horizontally throwing powder towards the plasma. The piezo actuated tray feeder is adapted to fit in this small space.

\subsection{Envelope}

The PMPI weight is $2.5 \mathrm{~kg}$, and uses the slower MPM transport speed, since it is not touching the plasma, getting to within $\sim 80-160 \mathrm{~mm}$ of the plasma edge. The only external connections possible are via electrical conductors through the MPM electrical connector probe mount.

\subsection{Magnetic Fields}

Using a magnetically driven motor was ruled out due to the 2.1 Tesla ambient magnetic field. Fast rotating conductive wheels with associated eddy currents were ruled out using high speed electric motors or air motors. Rotary feed-throughs were not possible due to the envelope and its location at the end of the probe electrical socket mount, preventing external mechanical drives. In addition, using an electrically conductive paddle wheel presents an inherent eddy current liability which could adversely affect operation. PEEK and stainless-steel paddles were tested with similar powder distributions. The PEEK paddles were used during initial operations and commissioning, based on permeability concerns $\left(>2.0 \mu / \mu_{0}\right)$ of the $316 /$ SS paddles being slowed by ambient field effects. The PEEK paddle wear will need replacement after every second load of boron carbide injection to keep the calibrated injection rate consistent.

A piezo motor is chosen [5], which can operate in the ambient 2.1T magnetic field, however its torque is low (50 mNm, @ $0.36 \mathrm{~Hz}$ rotation speed), ruling out a centrifugal force type injection, the paddle wheel is shown in figure 3 . Eight PEEK paddle arms $(.02 \mathrm{~mm}$ thick) are secured by set screws into the PEEK hub creating a paddle wheel diameter of $76 \mathrm{~mm}$, with $26 \mathrm{~mm}$ arms. The paddles bend slightly as they contact the aluminum delivery tray loaded with powder which causes them to spring free (spring constant is $2.8 \mathrm{~N} / \mathrm{m}$ ) accelerating powder horizontally forward out of the tray and through a hole in the carbon cover. The piezo motor supports the paddle wheel shaft on one end and the other is supported by a ceramic bearing [6].

The paddle wheel bench tests demonstrated a maximum throw of $600 \mathrm{~mm}$ with the highest density zone between 250 to $380 \mathrm{~mm}$ adequate to hit the plasma edge $150 \mathrm{~mm}$ away. The longer distances result in a lower thermal exposure for the injection time.

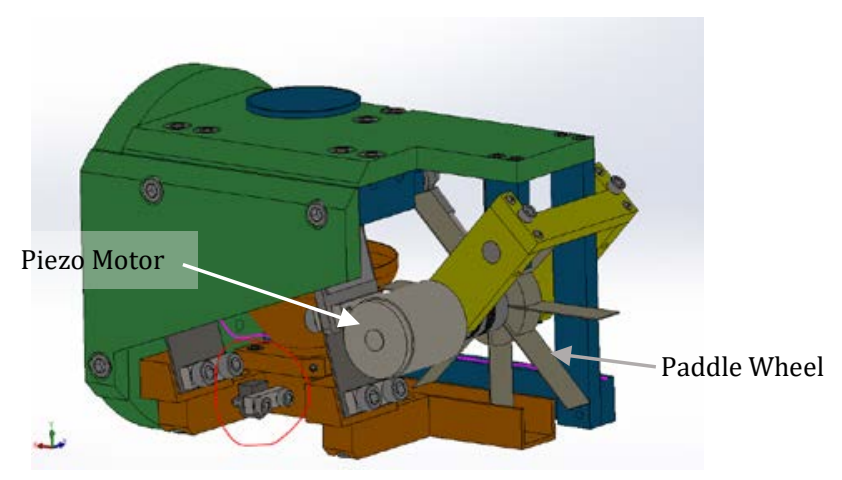

Fig. 3 The piezo motor direct drive through the paddle wheel axle, rotation switches and terminal blocks are not shown in this view for clarity.

\subsection{Powder Feeder}

A continuous layer of powder, 1-2 mm thick, is fed under the paddle wheel by a vibratory feeder, the design adapted from the piezoelectric feeder used on vertical powder droppers [3]. A side mounted tray with an $80 \mathrm{ml}$ volume cylindrical funnel/reservoir mounted at the opposite end of the paddle is vibrated causing powder to flow out of the $2 \mathrm{~mm}$ gap between the funnel output and delivery tray bottom toward the paddle wheel. The unit is operated at the tray structure resonant frequency $\sim 200$ Hz. The piezoelectric actuators are mounted at 10 degrees off vertical and move the powder upwards $\sim .1 \mathrm{~mm}$ and horizontal $\sim .3 \mathrm{~mm}$ maximum, towards the rotating powder wheel. A variable drive voltage using a signal generator/audio amplifier/transformer provides a voltage signal gain of 1:50 enabling calibrated operation for various powder flows. See Figure 4 


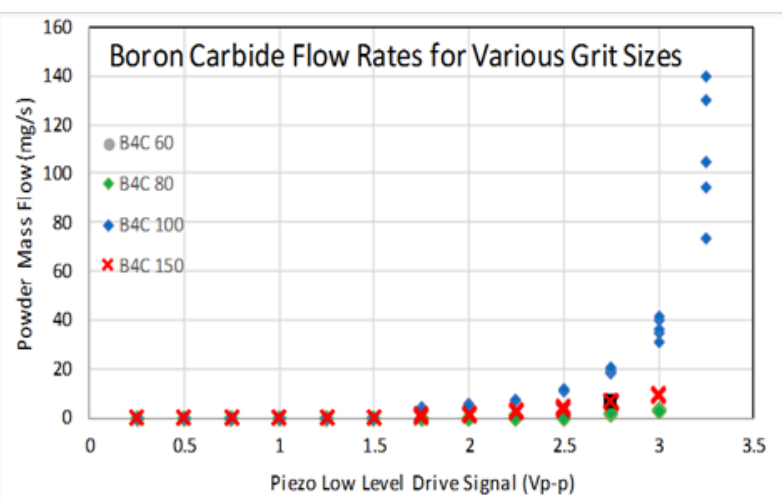

Fig. 4 The 60 grit material flowed more readily than the others, where the curves are one on top of each other, below $3 \mathrm{Vp}$-p drive voltage.

The unit contains two type $\mathrm{K}$ thermocouples located near the plasma end to monitor temperature, which must stay below 120C to keep the PEEK hub and paddles from deforming. A carbon cup, $6 \mathrm{~mm}$ wall, with a small aperture $13 \mathrm{~mm}$ wide $\mathrm{x} 20 \mathrm{~mm}$ high is cut in the plasma end across from the feeder tray allowing powder to pass through into the plasma. Approximately 50\% is injected while $50 \%$ is dropped into the carbon cup beneath the paddle wheel. The residue volume is approximately 80 $\mathrm{ml}$ and will allow 2 full reservoir loads before the residual in the cover must be emptied. The front view is shown below in Figure 5.

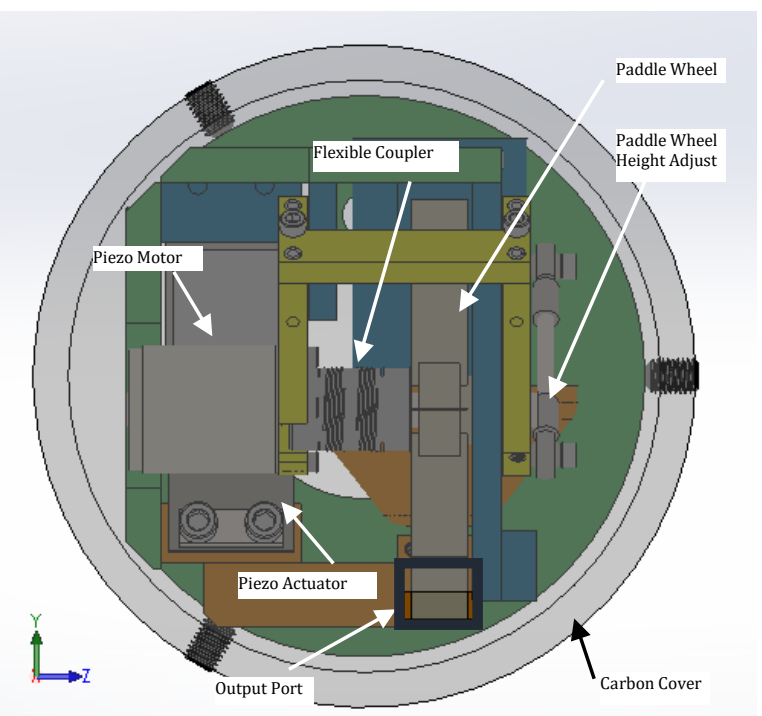

Fig. 5 The front view with carbon cover outlined.

\subsection{Operational Monitors}

Two cam-following micro-switches on the flexible shaft coupler detect piezo motor/paddle wheel rotation and direction. The motor is driven open loop and the two micro-switches ride on a cam cut into the piezo motor coupling to sense rotation and direction, although the motor is typically run a maximum speed.

The piezoelectric feeder resonant frequency is determined via accelerometer sensing the axial feeder tray accelerations and is detected without feeding powder by driving the unit at sub $50 \mathrm{Vp}-\mathrm{p}$ at the piezo. The resonance is determined by maximizing a peak voltage output value typically around $\sim 65 \mathrm{Vp}-\mathrm{p}$ (the bandwidth is $\sim 2-3 \mathrm{~Hz}$ wide). The resonant frequency is directly proportional to the system mass and can be checked and adjusted remotely between shots. A typical powder throw pattern is shown in figure 6 .

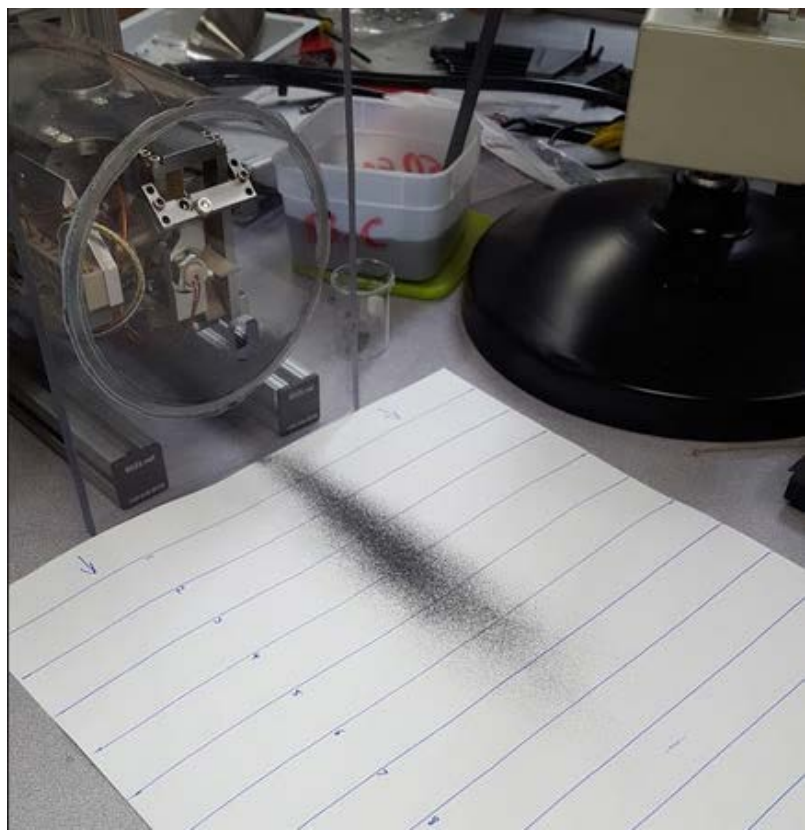

Fig. 6 The powder injection plume shown above, the lines are in inches, plume bulk peak is $\sim 100 \mathrm{~mm}$ and reaches $>160 \mathrm{~mm}$.

\subsection{Installation}

The defining envelope requires the unit to be lowered into the MPM chamber with a parallelogram fixture that can tilt the unit approximately 10-20 degrees to fit through the vertical installation port. The unit is then leveled, pushed into the electrical socket and the nut secured. The reservoir is then filled with powder, using a metal funnel and tube, through a hole in the carbon cup, into the reservoir. Metal fill components are used to eliminate any powder static charge buildup. A second refill once empty is achieved using the same technique without unit removal from the MPM. A third refill requires that the unit be remove and emptied of the noninjected powder that ends up dropping into the bottom of the carbon shield.

\subsection{Electrical Connections}

Electrical conductor needs are: Piezo actuators (2), Piezo motor leads (6), two thermocouples (4) and two microswitches (4). These are wired through an internal terminal block shown in figure $2 \mathrm{~b}$ which provides flexibility during testing and corrective maintenance tasks. The wires are insulated with vacuum rated $\left(10^{-6}\right.$ mbar) Kapton ${ }^{\circledR}$, Teflon ${ }^{\circledR}$, and aluminum oxide beads. 


\subsection{Operation}

The operational mode of this device is to push powder into W7-X plasma through a hole in a protective carbon cover at a controlled rate using the PEEK paddle spring force. This is accomplished by turning on the piezoelectric vibratory actuators, moving powder from the reservoir down the tray to the paddle wheel interface $\sim 1-2$ s. The paddle wheel is rotated simultaneously and pulses powder, via release of spring tension moving the powder in front of it through the injection port to the plasma edge during a shot. The device timing and pulse width is controlled by the plasma control system with the amplitude being set as desired. The injection pattern, shown in figure 7 , has a dense region at the center of a narrow width $\sim 20 \mathrm{~mm}$ that is caused by each paddle launching a powder like a shovel flinging dirt onto a flat surface with a horizontal motion. Powder delivery quantity is adjustable and directly proportional to the feeder drive voltage. The throw distance is adjusted by changing the radial length of paddle compression on the tray bottom via turn buckle. Typically though this adjustment is not used since the MPM can be positioned as needed relative to the plasma edge.

In figure 7 the powder pattern is shown using a high speed camera taken using the same drive settings used for the pattern in figure 7. There is a trail of less dense powder that extends back to the PMPI and will fall to the vessel floor and is estimated to be $\sim 10 \%$ of the injected volume.

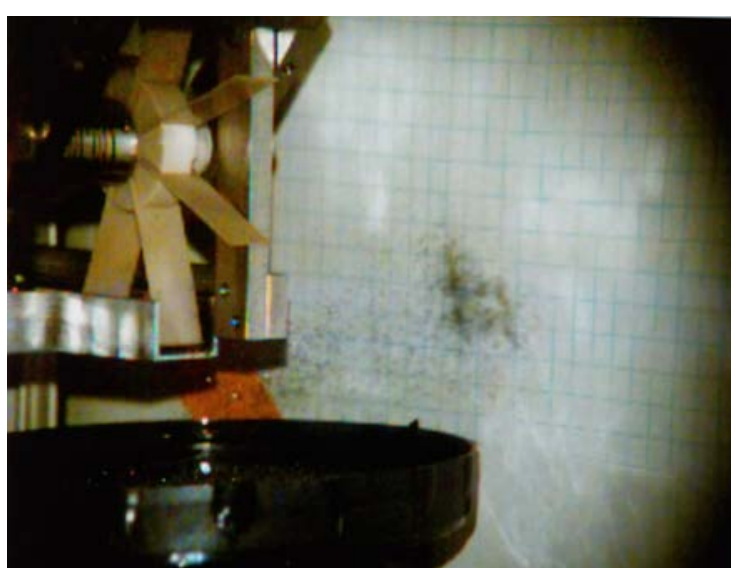

Fig. 7 Bench test launching of boron carbide powder is shown above. A packet of powder leads the less dense trailing powder indicating that a majority of that launched through the carbon cover opening will reach the plasma.

\subsection{Conclusion}

The kernel operational mode of this PMPI is the spring action of PEEK paddle arms against a vibrating powder delivery tray. The rest of the device is designed to support this behavior inside the prescribed envelope. The piezoelectric motor drive frequency $(.33 \mathrm{~Hz})$ is increased to $2.6 \mathrm{~Hz}$ using an eight-paddle wheel smoothing injection delivery. Eight paddles insure the leading paddle's shadow interference does not impinge on the following paddles powder delivery. All device material constraints are met to prevent any vacuum or magnetic limitations, due to the ambient environment. While this unit will not meet long term continuous operational needs, it does provide means for testing of multiple powders for plasma impurity injection research.

\section{Acknowledgments}

I thank David Mauzey, the main contributing design team member, student engineering intern, who brought this idea to life through his design work, fabrications, assembly, and testing.

This work has been supported by the U.S. Department of Energy under DE-AC02-09CH11466.

\section{References}

[1] Lunsford $\mathrm{R}$ et al., "Active conditioning of ASDEXUpgrade tungsten PFCs through boron particulate injection" Proc. 27th Int. Conf. in Fusion Energy 2018 ( Gandhinagar, India ) FIP/2-3

[2] A. Bortolon et al., Real-Time Wall Conditioning By Controlled Injection Of Boron And Boron Nitride Powder In Full Tungsten Wall ASDEX-Upgrade, Nuclear Materials and Energy, NME_2018_218

[3] A. Nagy et al., A Multi-Species Powder Dropper for Magnetic Fusion Applications, Review of Scientific Instruments 89, 10K121 (2018)

[4] D. Nicolai, et al., A Multi-Purpose Manipulator System for W7-X As User Facility For Plasma Edge Investigation, Fusion Engineering and Design 123, (2017) 960-964

[5] Piezo Motor Uppsala AB, Stalgatan 14, SE-754 50, Uppsala, Sweden

[6] Boca Bearing Company, 1420 Neptune Dr. Suite A, Boynton Beach, Fl, 33426 